\title{
Conservation of the gene structure and membrane- targeting signals of germ cell-specific lamin LIII in amphibians and fish
}

\author{
Helmut Hofemeister ${ }^{a}$, Cäcilia Kuhn ${ }^{\mathrm{b}}$, Werner W. Franke ${ }^{\mathrm{b}}$, Klaus Weber ${ }^{\mathrm{c}}$, Reimer Stick ${ }^{1) a}$ \\ a Department of Cell Biology, University of Bremen, Bremen/Germany \\ b Division of Cell Biology, German Cancer Research Center, Heidelberg/Germany \\ c Department of Biochemistry, Max Planck Institute for Biophysical Chemistry, Göttingen/Germany
}

Received August 17, 2001

Received in revised version November 12, 2001

Accepted November 25, 2001

\begin{abstract}
Nuclear lamina - lamin LIII - membrane-targeting motifs oocyte - Danio rerio
\end{abstract}

\begin{abstract}
Targeting of nuclear lamins to the inner nuclear membrane requires CaaX motif-dependent posttranslational isoprenylation and carboxyl methylation. We previously have shown that two variants of lamin LIII (i.e., LIIIa and LIIIb) in amphibian oocytes are generated by alternative splicing and differ greatly in their membrane association. An extra cysteine residue (as a potential palmitoylation site) and a basic cluster in conjunction with the CaaX motif function as secondary targeting signals responsible for stable membrane association of lamin LIIIb. cDNA sequencing and genomic analysis of the zebrafish Danio rerio lamin LIII uncovers a remarkable conservation of the genomic organization and of the two secondary membranetargeting signals in amphibians and fish. The expression pattern of lamin LIII genes is also conserved between amphibians and fish. Danio lamin LIII is expressed in diplotene oocytes. It is absent from male germ cells but is expressed in Sertoli cells of the testis. In addition, we provide sequence information of the entire coding sequence of zebrafish lamin $A$, which allows comparison of all major lamins from representatives of the four classes of vertebrates.
\end{abstract}

\section{Introduction}

The nuclear lamina is a filamentous meshwork associated with the nucleoplasmic surface of the inner nuclear membrane. In amphibian oocytes the organization of the lamina is particularly regular: 10-nm filaments form a near-tetragonal lattice, which harbors the nuclear pore complexes (Aebi et al., 1986). Lamins,

\footnotetext{
1) Dr. Reimer Stick, Department of Cell Biology, University of Bremen, PO Box 3304 40, D-28334 Bremen/Germany, e-mail: stick@uni-bremen.de, Fax: +494212189544.
}

members of the multigene family of intermediate filament (IF) proteins (Fuchs and Weber, 1994), are the major constituents of the lamina. They provide mechanical stability to the nuclear envelope and are involved in chromatin organization, DNA replication, and anchoring of nuclear pore complexes (Wilson et al., 2001). The importance of lamin function is highlighted by a growing number of mutants of the human gene encoding lamin $\mathrm{A} / \mathrm{C}$ that give rise to a variety of dominant congenital diseases (Hegele, 2000; Hutchison et al., 2001), by the targeted disruption of the lamin A/C in mice (Sullivan et al., 1999), the study of a Drosophila mutant with reduced lamin Dmo activity (Lenz-Böhme et al., 1997), and by RNAi experiments with Caenorhabditis (Liu et al., 2000).

Vertebrates express a variety of lamins that are encoded by separate genes or are generated by differential RNA splicing (Döring and Stick, 1990; Fisher et al., 1986; Furukawa and Hotta, 1993; Furukawa et al., 1994; Machiels et al., 1996). Two types of lamins, A- and B-type, can be distinguished based on their domain structure. The larger A lamins possess about 100 additional amino acid residues in their tail domain that are encoded by an extra exon (Lin and Worman, 1993; Stick, 1992). It has been suggested that A-type lamins arose from a B-type lamin ancestor by insertion of this additional exon during vertebrate evolution (Stick, 1992). A-type lamins have been isolated so far from mammals, birds and amphibians. They seem to be restricted to vertebrates since all molecularly characterized invertebrate lamins are of the B-type (Riemer et al., 2000). The lamin B1 and B2 genes are generally constitutively expressed in somatic cells of mammals and birds, while the synthesis of lamin A is developmentally regulated (Lehner et al., 1987; Röber et al., 1989; Stewart and Burke, 1987; Wolin et al., 1987). Amphibians synthesize a fourth lamin in addition to lamin A, B1, and B2, which has been named lamin LIII or lamin B3 (Benavente et al., 1985; Stick and Hausen, 1985; Stick and Krohne, 1982). The latter name was originally chosen to emphasize its molecular structure, which is of the B-type (Stick, 
1992). The name B3 has, however, also been given to a splice variant of mouse lamin B2, which specifically appears in male germ cells (Furukawa and Hotta, 1993). We, therefore, will stick to the name LIII for the sake of clarity.

Lamin LIII is the quantitatively major lamin of amphibian oocytes, eggs, and cleavage embryos (Benavente et al., 1985; Stick and Hausen, 1985; Stick and Krohne, 1982). In contrast to birds, which synthesize lamin A, B1, and B2 in diplotene oocytes (Lehner et al., 1987), B1 and B2 are very minor components in amphibian oocytes and lamin A is completely absent from these cells (Lourim et al., 1996; Wolin et al., 1987).

Lamins are postranslationally farnesylated and carboxylmethylated. These modifications depend on the presence of a C-terminal CaaX motif and are necessary for the association of lamins with the inner nuclear membrane (Holtz et al., 1989; Kitten and Nigg, 1991; Krohne et al., 1989; Loewinger and McKeon, 1988; Nigg et al., 1992). Lamin B1 and B2 are permanently isoprenylated and remain membrane associated after mitotic lamina disassembly (Gerace and Blobel, 1980; Hennekes and Nigg, 1994; Stick et al., 1988). Lamin A is proteolytically processed after incorporation into the lamina. This second maturation event removes the farnesylated and carboxyl-methylated cysteine residue of the processed CaaX motif (Beck et al., 1990; Kilic et al., 1999; Weber et al., 1989). This might explain why lamin A is selectively solubilized and dispersed during mitosis. Lamin LIII follows the roles of other B-type lamins. It is permanently isoprenylated and carboxylmethylated (Firmbach-Kraft and Stick, 1993). In contrast to lamins B1 and B2, the majority of lamin LIII becomes soluble in the course of nuclear envelope breakdown and only a minor fraction remains membrane associated (Lourim and Krohne, 1993). This phenomenon has been explained by the existence of two lamin LIII variants, a and b (Hofemeister et al., 2000), resulting from alternative splicing of lamin LIII transcripts and differing in their CaaX-encoding exons (Döring and Stick, 1990). While both proteins are farnesylated and carboxylmethylated (Firmbach-Kraft and Stick, 1993), the quantitatively minor variant LIIIb carries an extra cysteine residue, as a potential palmitoylation site, and a cluster of six basic amino acid residues in proximity of the CaaX box. Both motifs function in conjunction with CaaX modifications as secondary signals in membrane association (Hancock et al., 1990; Hofemeister et al., 2000).

The specific expression of the lamin LIII gene in amphibian oocytes and early embryos has been interpreted as an adaptation to the particular type of oogenesis and early development found in these organisms. Amphibian oocytes stockpile large amounts of maternal RNA and proteins during the long phase of oogenesis (Hausen et al., 1985). The volume of a Xenopus oocyte nucleus is about 100000 times that of a somatic cell nucleus (Hausen and Riebesell, 1991). The lamin present in the oocyte nuclear lamina serves as a pool for the generation of embryonic nuclei during the rapid cleavage divisions up to the 5000-10000 cell stage (Stick and Hausen, 1985). During this early phase of development cells are transcriptionally silent, protein synthesis solely depends on the recruitment of maternal mRNA, and the cell cycle is reduced to a S- and M-phase (Newport and Kirschner, 1982a).

Mammals and birds do not show such a rapid cleavage phase in their early development. Correspondingly, a lamin LIII homologue has not been found in these two classes of vertebrates, and databank search of the human genome did not provide evidence for an additional lamin gene (Hesse et al.,
2001). In contrast to mammals and birds, fish resemble amphibians in many respects of their oogenesis and early development (Kane, 1999). We therefore have searched for a homologue of the amphibian lamin LIII in the zebrafish Danio rerio, a model system of today's developmental biology.

Here we describe the sequence of lamin LIII of Danio rerio as well as its localization in the oocyte lamina by immunhistochemistry. Genomic analysis uncovers a remarkable conservation between the amphibian and the fish gene, suggesting that also in the fish two variants of lamin LIII are generated by alternative RNA splicing. Moreover, the secondary signals that are responsible for membrane association of Xenopus lamin LIIIb are perfectly conserved between amphibians and fish. In addition, we complete the sequence information for the major vertebrate lamins by providing the entire coding sequence of Danio rerio lamin A, thus allowing comparison of all major lamins from representatives of the four classes of vertebrates.

\section{Materials and methods}

\section{DNA techniques}

EST clones AW153707 and AW 421801 were obtained from Incyte Genomics (St. Louis, MO). Plasmids were purified using a plasmid purification kit (Qiagen, Hilden, Germany). Double stranded sequencing was done with internal primers using a Prism Dye Deoxy Terminator Cycle Sequencing kit (Applied Biosystems, Foster City, CA) and an ABI 392 DNA Sequencer. Since the Danio lamin A EST clones were not available at that time a DNA fragment covering the entire open reading frame was amplified by PCR. A cDNA library constructed from RNA of three-days-old Danio rerio embryos was used as template. This library was kindly provided by Holger Knaut (Max Planck Institute for Developmental Biology, Tübingen, Germany). PCR was carried out with sense primer 5'CCAGACAACCATGGAGACTC$\mathrm{CAGG}^{\prime}$ and antisense primer 5'CCAGGTCGACTTCGTCAACGGTGAC3' at $0.2 \mathrm{mM}$ in a $100-\mu$ reaction using TaqGold polymerase (Perkin Elmer-Cetus, Norwalk, CT) with the supplied buffer. Final concentration of $\mathrm{MgCl}_{2}$ was $10 \mathrm{mM}$. Cycling parameters were: initial denaturation $\left(5\right.$ minutes, $\left.95^{\circ} \mathrm{C}\right)$ followed by 35 cycles $(45$ seconds, $95^{\circ} \mathrm{C} ; 30$ seconds, $50^{\circ} \mathrm{C} ; 2$ minutes, $72^{\circ} \mathrm{C}$ ) and a final polymerization step $\left(10\right.$ minutes, $\left.72^{\circ} \mathrm{C}\right)$. The $\mathrm{PCR}$ product was separated on an agarose gel, purified using a gel extraction kit (Qiagen), and then directly sequenced.

\section{Antibodies}

Peptides corresponding to amino acids 2-16 (N-term: AMVTSTPMERSASAAC) and to amino acids 560-577 (C-term: PVRSRDAHMRRQGQTLDHC) of Danio rerio lamin LIII were synthesized by t-boc chemistry (Schnölzer et al., 1992) and coupled via a carboxyl-terminal cysteine residue to maleimide-activated keyhole limpet hemocyanin (KLH; $1 \mathrm{mg}$ peptide/1 mg KLH), using the Imject Activated Immunogen Conjugation kit (Pierce, Rockford, IL). Guinea pigs (two animals for each peptide) were immunized four times subcutaneously with $70-100 \mu \mathrm{g}$ protein per injection. For the first injection the KLH-coupled peptides were emulsified with an equal volume of complete Freund's adjuvant and, for booster injections at days 28,49 , and 70, with incomplete adjuvant. Blood was taken at day 86. Affinity purification of immunoglobulins was carried out with immobilized peptides. Peptides were coupled to SulfoLink (Pierce) with $1 \mathrm{mg}$ peptide per ml matrix. Coupling was carried out by Peptide Specialty Laboratories (Heidelberg, Germany). Five ml peptide-resin was used per $\mathrm{ml}$ of serum. The peptide-resin was first equilibrated with five volumes of PBS ( $\mathrm{pH} 7.4$ ) and then mixed for 1 hour at room temperature with one $\mathrm{ml}$ of serum diluted $1: 5$ in PBS. The flow through was collected, and the resin was washed with 20 volumes of PBS. Antibodies were 
eluted in fractions of $0.5 \mathrm{ml}$ with $0.2 \mathrm{M}$ glycine ( $\mathrm{pH} 2.5$ ) and immediately neutralized with $25-30 \mu \mathrm{l} 1 \mathrm{M}$ Tris- $\mathrm{HCl}(\mathrm{pH} 9.5)$. Purified antibodies, supplemented with an equal volume of glycerol, containing BSA at a final concentration of $0.3 \%$ and $0.025 \% \mathrm{NaN}_{3}$, were stored at $-20{ }^{\circ} \mathrm{C}$.

\section{Animals and histological procedures}

Wild-type zebrafish (Danio rerio) was raised and kept under standard laboratory conditions at about $28^{\circ} \mathrm{C}$ (Westerfield, 1995). Oocytes were staged as described (Selman et al., 1993). Tissues were frozen in isopentane cooled with liquid nitrogene to $-140^{\circ} \mathrm{C}$, and then stored at $-70^{\circ} \mathrm{C}$. Frozen tissues were embedded in Tissue-Tek (OCT 4583 embedding compound; Miles, Elkhart, IN) and frozen as described above. Tissues were sectioned $(4-5 \mu \mathrm{m})$, fixed with acetone for 10 minutes at $-20^{\circ} \mathrm{C}$, and then air-dried. Sections were blocked for 15 minutes with $1 \%$ BSA in PBS and incubated with appropriately diluted primary antibodies for 30 minutes, washed two times for 5 minutes, and incubated with $\mathrm{Cy} 3$-conjugated goat anti-guinea pig IgG (Dianova, Hamburg, Germany) for 30 minutes. After two washes for 5 minutes with PBS, sections were air-dried and mounted with Fluoromount-G (Southern Biotechnology Association, Birmingham, AL). Immunofluorescence was documented with an Axiophot photomicroscope (Zeiss, Jena, Germany) on T-max film (Kodak, Stuttgart, Germany). Films were scanned with a Coolscan 4000 scanner (Nikon, Tokyo, Japan) using SilverFast imaging software (LaserSoft Imaging, Kiel, Germany). Digital images were further processed with Photoshop (Adobe, San Jose, CA) and FreeHand (Macromedia, San Francisco, CA).

\section{Protein extraction, SDS-PAGE and immunoblotting}

A crude cytoskeletal preparation of zebrafish oocytes was prepared either from individual full-grown oocytes or small pieces of ovary. The tissue was homogenized using a tight-fitting, hand-driven homogenizer in an excess of extraction buffer $(1 \mathrm{M} \mathrm{NaCl}, 0.5 \%$ Triton X-100, $50 \mathrm{mM}$ Tris-HCl, $\mathrm{pH}$ 7.5). The cytoskeletal fraction was recovered by centrifugation for 10 minutes at $10000 \mathrm{~g}$. It was washed twice in extraction buffer and twice in distilled water, dissolved in SDS-sample buffer, and treated with $0.5 \mathrm{U} / \mu$ l Benzonase (Merck, Darmstadt, Germany) before boiling for 5 minutes. Samples were separated by 10\% SDS-PAGE. Material equivalent to about $5 \mathrm{mg}$ original tissue (wet weight) was loaded per lane. After electrophoresis polypeptides were transferred to Immobilon polyvinylidene difluoride (PVDF) membranes (Milipore, Bedford, MA). The transferred proteins were stained with Coomassie Blue. Immunodetection was as described (Huttenlauch et al., 1998).

\section{Results}

\section{Characterization of a homologue of the amphibian lamin LIII in fish}

Databanks were searched for a homologue of the amphibian lamin LIII. The entire protein sequence of Xenopus lamin LIII (Stick et al., 1988) (accession number CAA31567) was used as query in searches against the translated EST sequences of Danio rerio using the tBLASTN algorithm (Altschul et al., 1997). Among a number of hits with high scores to other vertebrate lamins, two sequences showed significant similarity to the N-terminal region of the query sequence. The inserts of these two clones (accession numbers AW 153707 and AW421801) were sequenced on both strands. Both cDNAs contained the complete open reading frame encoding Danio rerio lamin LIII. The two sequences differ in 12 nucleotide positions resulting in four amino acid exchanges. It is therefore reasonable that the two clones represent two different alleles of the same gene.

The conceptual translation product (Fig. 1) shows all features diagnostic for a vertebrate lamin protein (Fuchs and Weber, 1994), notably the long-coil 1 b version in the central rod domain typical for lamins and IF proteins from protostomic phyla. Two p34 ${ }^{\text {cdc2 }}$ protein kinase phosphorylation consensus sites (amino acids 31 and 401) flank the rod domain. These sequences are

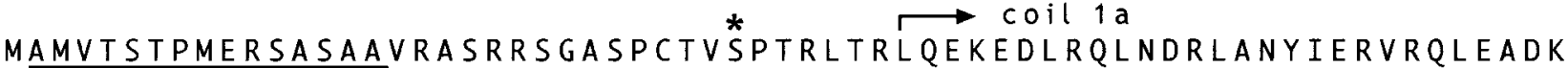

$\longleftrightarrow$ COII $1 \mathrm{~b}$

SSMQILLEEKEESSLREVGNVRRLYESELADARKSLDATANERARLQIEFSQLQEDHRKLAARNN 130

KKECELTAAVDRWRNLEVALNAKEADFANLLSTNRRLETEISELKTQVPALEAALQKVKTQLNSE 195

MLRRVDAENQIQTLQEQLDFQKHLGEQEVREMRRHESR

HETQIRQYKDELEKTFNAKLENAKQTAVKNSDFAFSTKEELAGTKLRLESQTQQINNLQKQVVCL 325

EARVLELQQTLEHERQVSQQKLSLQQQEMAALRLQMQEQLEEYENLLDVKLMLDMEINAYRKMLE 390 $\stackrel{2}{\rightleftarrows}$ GEEKRLNQSL
SPIQQTLSPIIQQSRKKRKHDATCSGFSPNYRLSQHSSCR GPVSIEEVDLDGRYI 455 KLKNASDRDQVVSGWQLRKSEAEAPDIVFCFPSPCVMNAGHMLTVWAAAAVDQCGSASDLLLSTH 520 QSWGSFADVRVSLLNTQNEEMAEYYVVQDKTEEDAESDEPVRSRDAHMRRQGQTLDHDSACLLM

Fig. 1. Amino acid sequence of Danio rerio lamin LIII as deduced from the nucleotide sequence of EST clone GenBank accession number AW153707. The subdomains of the central rod (coils 1a, 1b, and 2) are marked by arrows. The two p34 $4^{\text {cdc2 }}$ protein kinase sites, that flank the rod domain, are marked by asterisks. The nuclear localization signal is underlined by a broken line. The 105 -box within the tail domain is shown in brackets. The two peptides that were chosen for the generation of anti-peptide antibodies are underlined. The nucleotide sequence data are available from GenBank under the accession number AF397015. 
involved in the reversible dissociation of lamin filaments during cell division (Heald and McKeon, 1990; Peter et al., 1990). A canonical NLS is present within the first part of the tail domain (amino acids 415-419), as well as a conserved region of about 105 amino acid residues, the 105-box, within the tail domain (amino acids $441-539$ ) (Riemer et al., 2000), and a CaaX motif at the C-terminus. Danio LIII shows the greatest sequence drift among all vertebrate lamins and even in regions that are highly conserved between other lamins, as for example the 105-box within the tail domain, the Danio LIII sequences show significant sequence deviation. Danio lamin LIII lacks the extension of the tail domain that is characteristic of A-type lamins and can therefore be classified as a B-type lamin (Stick, 1992). Moreover, sequence comparison with mammalian, avian, amphibian, and fish lamins A, B1, B2 and with amphibian lamin LIII has revealed the closest sequence similarity to the latter, indicating that Danio lamin LIII is the homologue of Xenopus lamin LIII (see Fig. 6).

The amphibian lamin LIII is the major lamin of oocytes and early embryos (Stick and Hausen, 1985; Stick and Krohne, 1982). In other tissues this lamin occurs only in very few cells, as for example in Sertoli cells of the testis and in certain cells of neuronal and cardiac tissue (Benavente et al., 1985). To see whether the Danio lamin LIII gene is expressed in a similar pattern we have raised peptide antibodies that specifically recognize this lamin. Peptides for immunization were chosen from the $\mathrm{N}$-terminus and the $\mathrm{C}$-terminus, respectively, since these regions differ in sequence from all other three Danio lamins and are probably accessible to antibodies in the context of the entire protein. Two guinea pigs were immunized with each of the two peptides. All of the resulting four sera reacted strongly with the oocyte nuclear envelope on cryosections. In addition, these sera showed various but significant reactions with the vitelline envelope surrounding the oocytes. To clarify, whether this was due to cross-reaction with the lamins, LIIIspecific antibodies were isolated by affinity purification using the peptides conjugated to an affinity matrix. The affinitypurified antibodies immunostained exclusively the oocyte nuclear envelopes (Fig. 2A, D) while staining of the vitelline envelope was reduced to background level. Staining of sections with the flow through fraction demonstrated the efficiency of the affinity purification. While the non-bound antibodies decorated the vitelline envelope to a similar degree as the unfractionated serum, the reactivity against the oocyte nuclear envelopes was completely abolished upon affinity purification (Fig. 2G). None of the antibodies reacted with nuclei of follicle cells or other somatic cells present in the ovary (Fig. 2, compare DNA staining in B, E with immune staining in A, D). The sera were also tested on cryosections of fish testis. Significantly, one of the antibodies stained nuclei of Sertoli cells (Fig. 2J), reminiscent of the situation reported for certain anti-Xenopus lamin LIII antibodies (Benavente et al., 1985). All other tissues tested, such as liver, skeletal muscle, and kidney were negative (not shown). As expected from the sequence comparison the sera showed no cross-reactivity with Xenopus lamins (not shown).

The affinity-purified antibodies were also used to detect lamin LIII on immunoblots. Lamina-enriched fractions were obtained by extraction of oocytes or entire ovaries with a buffer containing $1 \mathrm{M} \mathrm{NaCl}$ and $0.5 \%$ Triton X-100. The resulting cytoskeletal fractions were separated by SDS-PAGE and blots were probed with affinity-purified antibodies. A single band of $66 \mathrm{kD}$ was detected with an antibody preparation directed against the C-terminal peptide (Fig. 3). The size of the reactive band corresponded well with the calculated molecular mass of $67063 \mathrm{Da}$ of the conceptual lamin LIII polypeptide.

\section{Conservation of the genomic organization between lamin LIII genes of fish and amphibians}

The Xenopus LIII gene gives rise to two mRNAs that are generated by alternative splicing. The resulting proteins, LIIIa and LIIIb, differ in their last CaaX-containing exon (Döring and Stick, 1990). These two lamin variants differ greatly in their membrane association. An extra cysteine as a potential palmitoylation site and a basic cluster is present in variant LIIIb. Both sequences function as secondary targeting signals in conjunction with the CaaX motif for the stable association of lamin LIIIb with membranes (Hofemeister et al., 2000).

To clarify whether this feature of LIII is conserved between amphibians and fish we have amplified the genomic region of the Danio LIII gene that corresponds to intron 11 and the flanking exon regions of the Xenopus LIII gene. A genomic fragment of about 1850-1900 nucleotides was amplified by PCR that corresponded to about 1700-1750 nucleotides of intron sequence. The fragment was sub-cloned and sequenced from both ends. We obtained sequence information of 813 nucleotides from the $5^{\prime}$-end and 1032 nucleotides from the $3^{\prime}$ end that covered nearly the entire fragment (Fig. 4B). Since sequencing reactions from both ends terminated at the same positions under a variety of reaction conditions, we were unable to obtain an overlap of the two sequences. The 1032 nucleotides of the 3 '-end, however, contained relevant information. It contained the sequences corresponding to the cDNA sequence of clone AW153707 as well as a short exon (exon 11b in Fig. 4A) encoding an amino acid sequence that closely resembled that of exon $11 \mathrm{~b}$ of the LIII gene of Xenopus. The intron/exon structure of this region was identical to that of the homologue Xenopus gene, while, as expected, the actual sequences within the introns were not conserved (Fig. 4B) (Döring and Stick, 1990). Significantly, Danio exon 11 b encodes a polybasic cluster flanked by an extra cysteine residue and a CaaX box. The sequence of the basic cluster is perfectly conserved between Danio and Xenopus and the CaaX sequence deviates only in one of the three variable residues (Fig. 4C). Taken together, this conservation strongly suggests that in Danio, like in Xenopus, two lamin LIII variants are generated by alternative splicing. This finding points to a specialized function that this lamin variant might fulfill during oogenesis and/or early development in amphibians and fish.

\section{Sequence of lamin A of Danio rerio}

Sequence information for the three lamin sub-types, A, B1, and $\mathrm{B} 2$ is available from at least one representative of the four classes of vertebrates, with the exception of lamin A from fish. Since it is widely assumed that A-type lamins evolved during vertebrate evolution we have been particularly interested to see whether lamin A is present in fish.

We therefore have searched the Danio EST data bank with either the Xenopus lamin A sequence (Wolin et al., 1987) or the lamin LIII sequence (Stick et al., 1988) as query. Several EST sequences were found that encode Danio lamin A. The ESTs contain sequences either from the $5^{\prime}$ - or the $3^{\prime}$-end of the coding region (accession numbers BE201026, BE557685). Since the corresponding cDNA clones have not been available at that time we have amplified the entire coding region by PCR, using 

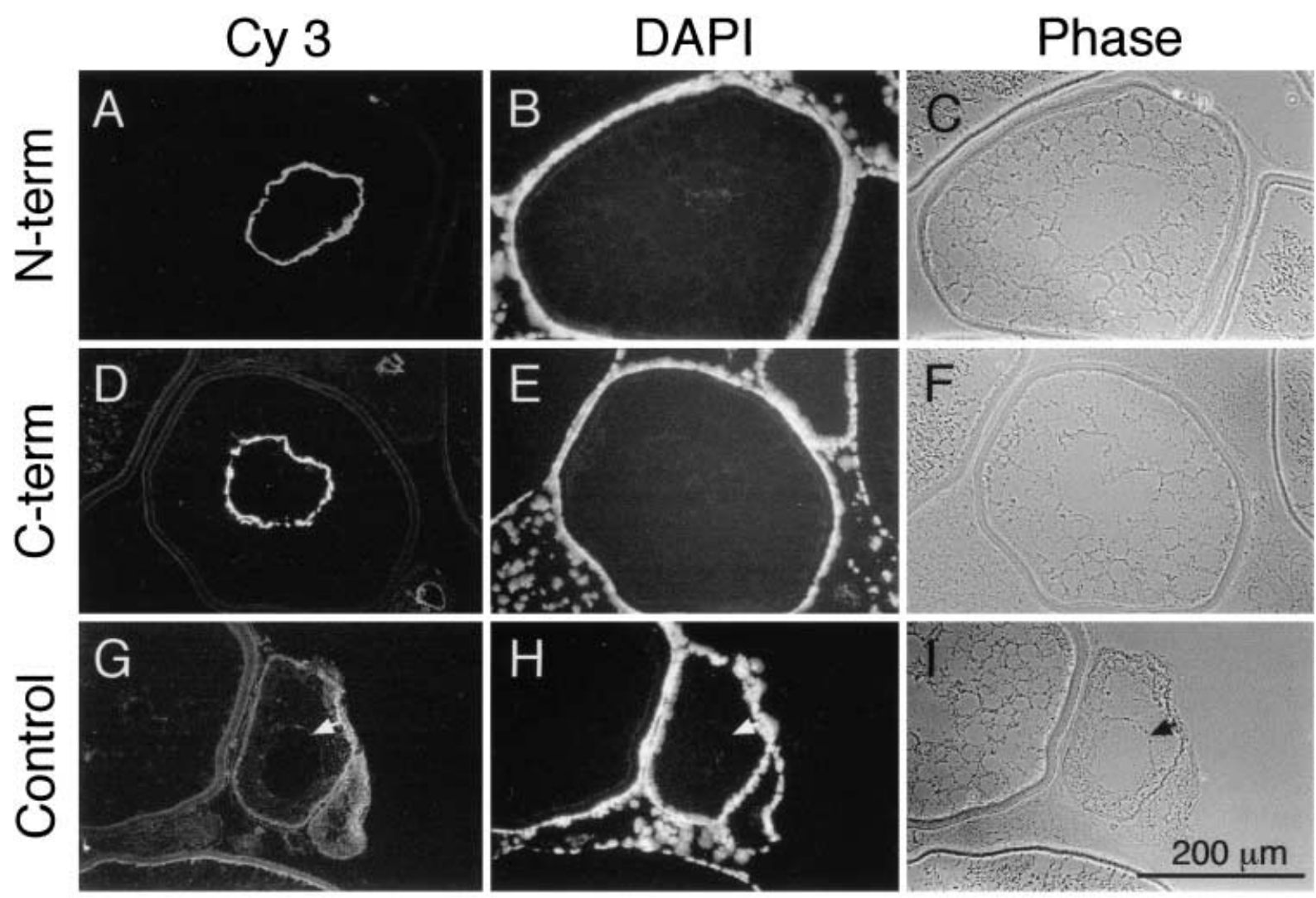

Cy 3

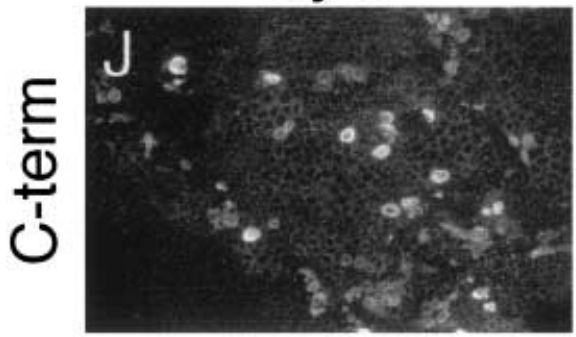

Phase

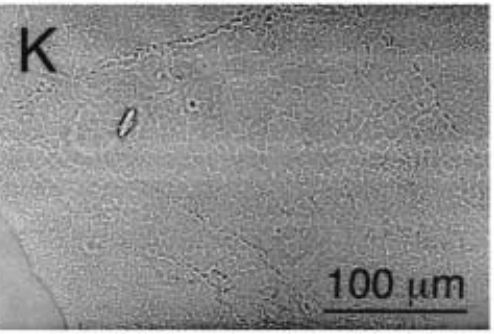

Fig. 2. Immunolocalization of lamin LIII in zebrafish ovary and testis. Cryosections (4-5 $\mu \mathrm{m})$ of Danio rerio ovary $(\mathbf{A}-\mathbf{I})$ and testis $(\mathbf{J}, \mathbf{K})$ were fixed with cold acetone and stained by indirect immunofluorescence with affinity-selected anti-peptide antibodies directed against an N-terminal peptide of Danio lamin LIII (N-term: A), a C-terminal peptide (C-term: $\mathbf{D}, \mathbf{J})$, or with the flow through fraction of the affinity purification of an antiserum directed against the C-terminal peptide (Control: G). Sections were counterstained with diamidinophenylin-

as template oligo-dT-primed cDNA from 3-days-old embryos, anticipating that expression of Danio lamin A begins only at later stages of development, similar to the situation in other vertebrates. After gel purification the PCR product was directly sequenced in its entire length using internal primers. The conceptual translation product showed all features diagnostic for vertebrate lamins as explained above for lamin LIII (Fig. 5). It contained an extra domain of 71 amino acid residues (position 578-648) in its tail domain, the hallmark of A-type lamins (Fisher et al., 1986; Fuchs and Weber, 1994) (Fig. 5). Danio lamin A showed the largest sequence divergence of all A-type lamins. Interestingly, pairwise sequence alignment has revealed, that the LIII lamins are closer to A-type than to the other B-type lamins. dole to visualize the position of nuclei (DAPI: B, E, H). Resolution of follicle cell nuclei is somewhat limited since acetone fixation, which was chosen to optimally maintain immuno-reactivity, does not fully preserve nucleic acids. Note that the concentration of DNA in the giant oocyte nuclei is too low to be detected under the conditions used here. Arrows in $(\mathbf{G}, \mathbf{H}$, and $\mathbf{I})$ indicate the position of the nuclear envelope. The phase-contrast micrographs in $(\mathbf{C}, \mathbf{F}, \mathbf{I}$, and $\mathbf{K})$ correspond to frames $(\mathbf{A} / \mathbf{B}, \mathbf{D} / \mathbf{E}, \mathbf{G} / \mathbf{H}$, and $\mathbf{J})$, respectively.

The intron positions of several lamin genes have been mapped (Döring and Stick, 1990; Lin and Worman, 1993, 1995; Zewe et al., 1991). They are highly conserved. In the course of our EST search for Danio lamin A we have also detected a sequence tag (accession number BE016225) containing an 82 nucleotides long intron. Comparison with other Danio ESTs and with genes encoding the Xenopus and the human lamin A genes have revealed that this intron is the homologue to intron 8 in the amphibian and human gene (Lin and Worman, 1993; Stick, 1992). Its position is perfectly conserved in the Danio gene. 


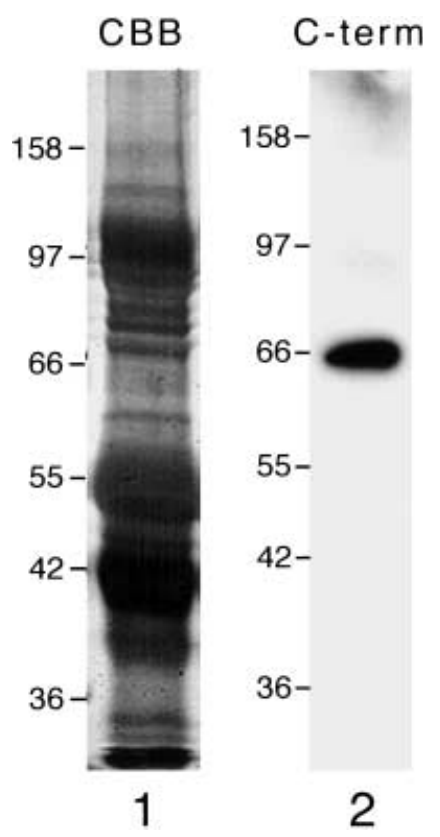

Fig. 3. Analysis of zebrafish lamin LIII by immunoblotting. Fullgrown Danio rerio oocytes were extracted with a buffer containing high salt and non-ionic detergent, and the insoluble fraction was separated by $10 \%$ SDS-PAGE. Polypeptides were transferred to PVDF membranes and either stained with Coomassie Blue, lane 1 (CBB) or probed with affinity-selected antibodies directed against a C-terminal peptide of Danio rerio lamin LIII, lane 2 (C-term). The relative mobility of marker proteins maltose-binding protein- $\beta$-galactosidase fusion protein $(158 \mathrm{kDa})$, phosphorylase $\mathrm{b}(97 \mathrm{kDa})$, bovine serum albumin $(66 \mathrm{kDa})$, glutamic dehydrogenase $(55 \mathrm{kDa})$, maltose-binding protein $(42 \mathrm{kDa})$, and lactate dehydrogenase $\mathrm{M}(36 \mathrm{kDa})$ are indicated.

\section{Discussion}

We have identified lamin A and a homologue of the amphibian lamin LIII in the zebrafish, Danio rerio, by searching the Danio EST data bank. By providing the complete sequences of the two fish lamin polypeptides we complete the catalog of vertebrate lamin sequences. Classification of the two fish sequences was based on overall sequence similarity to the other known vertebrate lamins. The presence of an extra domain within the tail of Danio lamin A is diagnostic for A-type lamins and allowed an unambiguous identification of this protein as an Atype lamin (Fuchs and Weber, 1994; Lin and Worman, 1993; Stick, 1992). A-type lamins form a relatively homogenous group of lamins that seems to be restricted to vertebrates. The presence of lamin $\mathrm{A}$ in fish strengthens the view that A-type lamins have been acquired at the base of the vertebrate lineage. Their expression is developmentally controlled, and they are absent from undifferentiated cells (Lehner et al., 1987; Röber et al., 1989; Stewart and Burke, 1987). In mammals, the lamin A gene gives rise to several variants by differential splicing which points to a role in cell differentiation (Fisher et al., 1986; Furukawa et al., 1994; Machiels et al., 1996).

The sequence of lamin LIII from a second vertebrate class now allows positioning of this type of lamin within the lamin family: Danio lamin LIII shows the highest sequence similarity to Xenopus LIII but similarity values to A-type lamins are also high. However, sequence similarity between Xenopus LIII and several A-type lamins is even higher than between lamin LIII of
Xenopus and fish. While our study was underway, Nahagama and colleagues cloned the goldfish lamin LIII (B3) (Yamaguchi et al., 2001), and identified the major polypeptide of the germinal vesicle lamina as lamin LIII by peptide sequence analysis and by cDNA sequencing.

Sequence identity between zebrafish and the goldfish sequences is about $70 \%$ while identity values drop to about $50 \%$ between the fish and the Xenopus sequences. Homologues of lamin LIII have not yet been identified in birds and mammals, and data bank search of the human genome has not provided evidence for an additional lamin gene (Hesse et al., 2001). Despite the large sequence drift and together with the evidence presented below we conclude that fish and amphibians contain and express an additional lamin gene, LIII, representing a distinct type (Fig. 6). Obviously, LIII lamins display the general features of B-type lamins, but on the other hand show the highest sequence similarity to A-type lamins. The high sequence drift between the three known LIII sequences is remarkable and suggests that these lamins represent an ancient form of (vertebrate) lamin serving functions common to fish and amphibians.

\section{LIII lamin genes show a common expression pattern in fish and amphibians}

Expression of the lamin LIII gene has been studied in particular detail in Xenopus, where it is the major lamin of oocytes and early embryos. In the ovary, it is expressed in diplotene oocytes. It is absent from male germ cells but is expressed in Sertoli cells of the testis as well as in a few cells in somatic tissues (Benavente et al., 1985; Stick and Hausen, 1985; Stick and Krohne, 1982). Moreover, in the present study the synthesis of Danio lamin LIII has been examined with antibodies that recognize peptide sequences unique to Danio lamin LIII. Danio lamin LIII is exclusively expressed in diplotene oocytes in the ovary and in Sertoli cells of the testis. In both tissues the expression pattern matches exactly that described for amphibian lamin LIII. Moreover, in Xenopus as well as in goldfish, LIII is the quantitatively major lamin of oocytes (Lourim et al., 1996; Stick and Krohne, 1982; Yamaguchi et al., 2001). These observations give further support to the view that these lamins are homologous. In contrast, birds synthesize all three lamin sub-types, B1, B2, and lamin A in diplotene oocytes (Lehner et al., 1987). We therefore assume that LIII serves a specialized function in fish and amphibians that is absent in other vertebrate classes.

\section{Conservation of lamin LIII splice variants}

The most compelling evidence for a common function of lamin LIII in amphibians and fish is the striking conservation of their CaaX motif-encoding exons. Two variants of lamin LIII are expressed in Xenopus (Döring and Stick, 1990), generated by alternative splicing of two C-terminal CaaX motif-encoding exons. The two variants differ greatly in their behavior during meiotic nuclear envelope breakdown: LIIIb remains selectively associated with membranes upon lamina disassembly (Hofemeister et al., 2000). Mutational analysis has shown that a cluster of six basic amino acids and a cysteine as a potential palmitoylation site is necessary for stable association of LIIIb with membranes. In contrast, lamin LIIIa, that lacks these secondary signals, becomes soluble during nuclear envelope breakdown. The genomic organization of the two alternative exons within the LIII gene is perfectly conserved between Xenopus and Danio. Even more strikingly, the actual sequence 
A

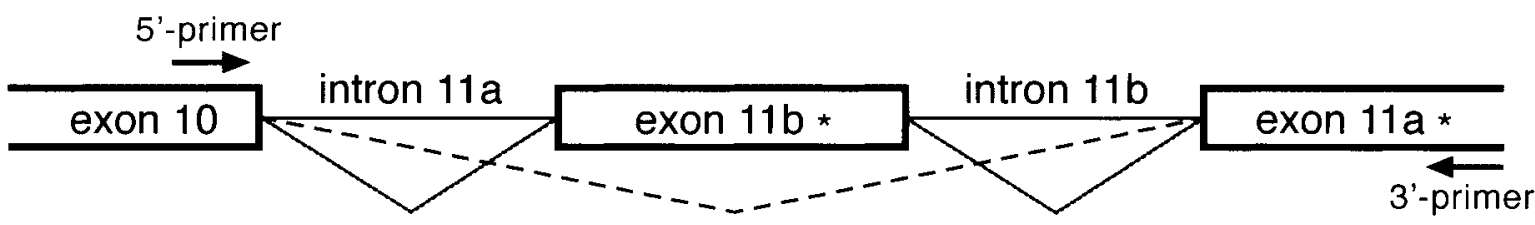

B

$\begin{array}{llllllllllllll}E & P & V & R & S & R & D & A & H & M & R & R & Q\end{array}$

GAA CCG GTG AGG AGT CGC GAC GCT CAC ATG CGC AGA CAG gtaacacacaccacacacacacactcttta

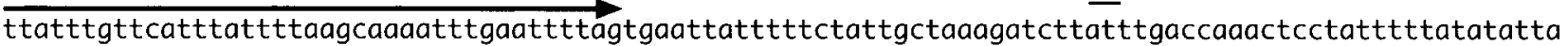
aataaatgaccaaactctttattttgatatatgcgtgtagaatatggatgttttagactatctaaaggtgtactcacactatgctatctgaaccgtgc tcaggcccatttcccggatcgtttgaggagtgtgagtgctctgaatcgggctcaggcatggttcagttggctgtccatagcccggttagaagaagtgt gccagagcgcggttcactttgggctttggcgcggtacgcttgtgtgacttttaaggcactgtttgatctggatttattaatcattcttattgttcaat caacacaaactgtggtagattattaaagacacaaacccctcactgcagcacagctgcaccttcagcagacctcctcatacctgcagcagcaggactcc atgattatgaagtggctgatctgttcagcagaatatttgactgtgtcactgcatatcaaaccactaaacccatataactaaagaatgtccactgtgcc tcagcccagttcaaggtgactggacatagtgtgagtacacctaacacaggttgagttttactgtcagtctcaatcatcttcaacatcagtgaacgca tgaaccagagcaggtaaagctggtcgtttctctgaagcacaggtgtcagttccagttc...

... cacctgtgctccagataaa cacgtctgccagatgcataacatcagagattgtctgtcttcttcatctgtcttttataaaag

$\begin{array}{lllllllllllll}A & K & R & R & K & K & K & C & C & F & V & S\end{array} *$

GCC AAA AGG AGG AAA AAG AAG TGT TGT TTT GTG TCC TGA agactagtgtctgtaggtgaggagacgtca gtctgtctgcagcgcttgtgctttgcttgttaacatcagcttagcatgtttgctaacactggacatcatcaggaacatattattattattatcattt attctgattctaatgctgttattaatttaccagaacaagctgcagtgtgcaagtgtttgcatgtcggataattgatgagacgtcgagttcttatagaa taatgcgctccgcatgtaaggaagtgtgcagtatagtttaataatccaccaacctgcaggtgatcactgctctcctcctcttgtcctgaatccactgt tttcaggtgcgtgtccctccacatctcctcctcctgatgttggtgtacctgatgtgatttctgacctgttgtagctgtgaagtcattcagcggaatga tatggaagctgtaatgcgctctacacccacctgagagtgaccatcagccaatcagaatatagcactatgttagtgtagtgatctaaagctaaacagcg ctctaaacctgccggaactactttagctgtgcctttattctgacaataaccaaacacctaagagtgaccatcagccaatcagaatgcagcactcagca gagctgtgggtaaatgttagtgtagtgatatgaagctgtaataaggcctaaacctgccggaactacttttgagagatctacaaataaacattaatcta aatattgaagtgtttataatgttgtgtgtgcgtgtgtgcgtgtgtgcgtgtgtgcgtgtgtgtgtgtgtgtgtgtttcag

$\begin{array}{lllllllllllllllll}G & Q & T & L & D & H & D & S & A & C & L & L & M & *\end{array}$ GGT CAG ACG TTG GAT CAT GAC TCT GCT TGT CTG CTG ATG TGA atctctgcgctgctcteatatctg aatgttaatgtttgtatttaatgaatgttcacggcctgacctggattg

C

$\begin{array}{ll}\text { Xenopus } & \text { LIIIa } \\ \text { Danio } & \text { LIIIa } \\ \text { Xenopus } & \text { LIIIb } \\ \text { Danio } & \text { LIIIb }\end{array}$

Fig. 4. Genomic organization and nucleotide sequence of the $3^{\prime}$-end of the coding region of the Danio rerio lamin LIII gene. Schematic representation of the exon/intron structure of the zebrafish lamin LIII gene $(\mathbf{A})$. Numbering of exons and introns is according to the homolog gene of Xenopus (Döring and Stick, 1990). Splicing of introns 11a and $11 \mathrm{~b}$, giving rise to an mRNA encoding lamin variant LIIIb, is indicated by dotted lines and is inferred from analysis of the Xenopus lamin LIII gene. The positions of primers that were used for the amplification of the genomic fragment are shown by arrows. (B) Nucleotide sequence and predicted amino acid sequence of the genomic region covering the

of the LIIIb-specific exon, exon $11 \mathrm{~b}$, is highly conserved. It differs in only one of the two variable positions within the CaaX motif (Fig. 4C). In contrast, sequence conservation of the LIIIaspecific exon is limited to only one residue outside the CaaX motif. This suggests that also in the fish two variants of lamin LIII are expressed. A direct proof that the two proteins are indeed expressed in Danio would require variant-specific
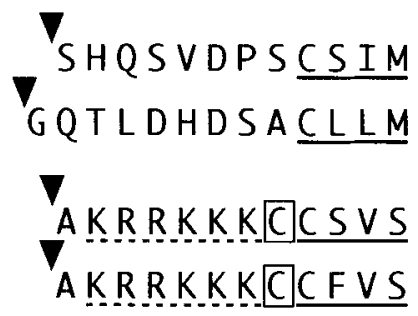

3 '-end of the coding region of the Danio rerio lamin LIII gene. Stop codons are marked by asterisks, the splice consensus sequences at the ends of the introns are underlined, the primer sequences used for amplifications are indicated by arrows. (C) Comparison of the amino acid sequences encoded by the two alternatively spliced exons of the Xenopus lamin LIII gene with the corresponding sequences of the Danio gene. Note the high conservation between the two LIIIb-specific sequences. The CaaX motifs are underlined, the extra cysteine residues, as potential palmitoylation sites, are boxed, and the polybasic clusters are underlined by broken lines.

antibodies. Generation of lamin LIIIb-specific antibodies, however, poses a major problem. It is hampered by the fact that the processed forms of the two proteins differ only by eight amino acid residues. One of the eight residues of LIIIb, the extra cysteine residue, will be modified in vivo and will therefore have altered antigenic properties. Another six residues form a cluster of basic amino acids that is similar or 


\section{* $\longrightarrow$ coil $1 \mathrm{a}$}

METPGQKRSSRGGVTNVLSPTRISRLQEKEDLSNLNDRLAVYIDKVRSLEVENAGLRMRITESET 65

$\longrightarrow$ COII $1 \mathrm{~b}$

EISRELSGMKAAYEAELADARKTLDSVAKERARLQLELSKVREDYKELKARNGKKEADLESALAR 130

LKDLESLLNSKDASLSTALGEKRTLEVEVRDLKAQLAKLEGSLNDAKKQLQDEMLRRVDAENRIQ 195

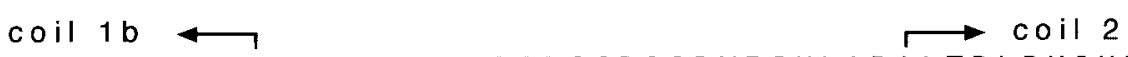

TLKEELEFQKNIYSEELRESKRRYESRVVEIDSGRQQDYESKLADALTDLRNQHEEQLRIYKEEI 260

EKTYNSKLENARSSAERNSHLVGAAHEELQQTRVRMEGVSSQLSQLQKQLAAREAKIRELEEALS 325

RERDILRRRLEDKEKEMAEMRQRMQQQLDEYQELLDIKLALDMEISAYRKLLEGEEERLRLSPSP 390

PPARGVTVTRSSGSGSHTRVVQSSTSRTSSGSAKKRRLNDNDSDASSVVGGTVTRTRIFQQASAS 455

$105-\mathrm{box} \rightarrow$

GRVTVDEVDLEGKFVRLNNKSDQDQSLGHWQVKRQIGSGTPIVYKFPPKFNLKAGQTVTIWAAGA 520

$\longleftarrow 105-b o x \quad$ VIamin A-

GGTHSPPSDLVWKTQNSWGSGDLFQTTLISSSGE]EMAMRKVTRTLFQDEEDDEMAAHSTCGDSEY 585

domain $\rightarrow$

$\leftarrow$ Iamin $A$ domain 7

NLRSRTVLCGSCGQPSDRNSSCVSASSGVSSASRSFSSGGGGGLTEAFVSPSHFIVSNDKPRQAG 650

\section{PKVDNCSIM}

Fig. 5. Amino acid sequence of Danio rerio lamin A as deduced from the nucleotide sequence of a cDNA clone obtained by PCR amplification using cDNA from three-days-old embryos (for details see Materials and methods). The subdomains of the central rod (coils 1a, $1 \mathrm{~b}$, and 2 ) are marked by arrows. The two $\mathrm{p} 34^{\mathrm{cdc} 2}$ protein kinase sites, that flank the rod domain, are marked by an asterisk. The nuclear

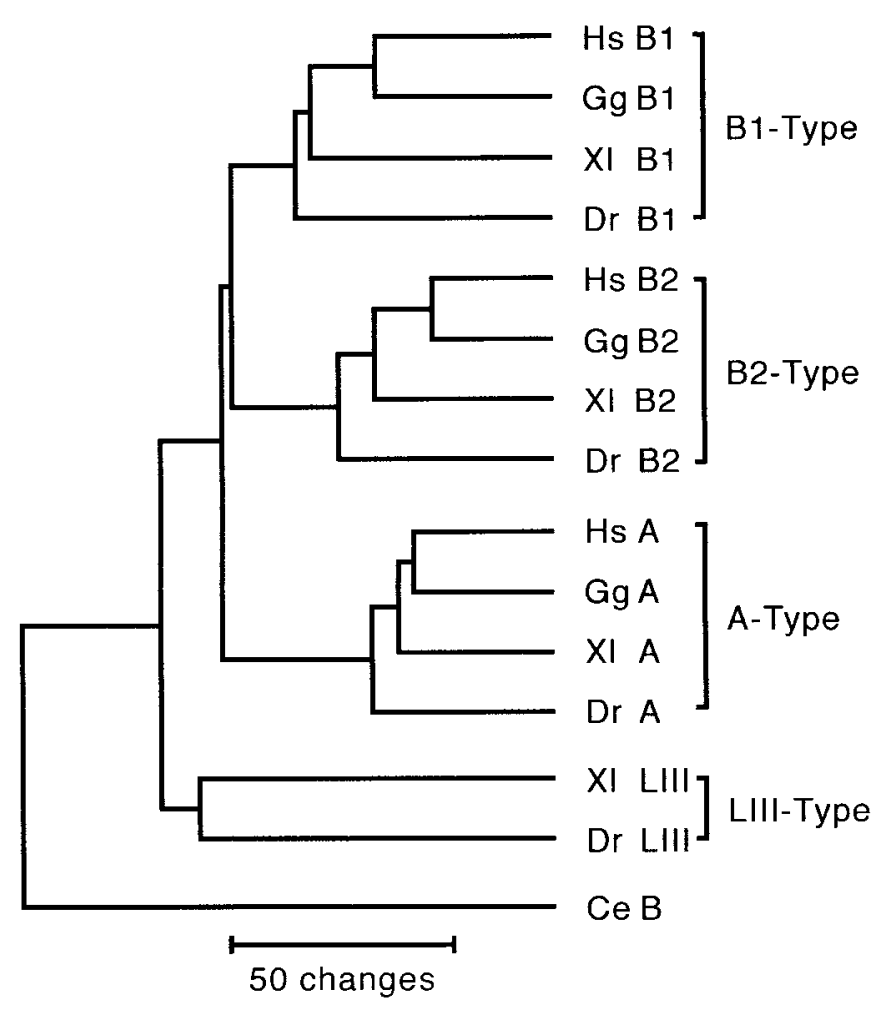

even identical to the basic clusters constituting the classical nuclear localization signals. We have previously demonstrated that the basic cluster of Xenopus lamin LIIIb, which is identical to that of the zebrafish, can indeed function as an NLS (Hofemeister et al, 2000). Antibodies raised against the lamin LIIIb-specific peptide sequence, if obtained at all, would almost certainly recognize a wide variety of NLS-containing proteins making these antibodies useless as LIII-specific reagents.

The strict conservation of the sequence of the six basic residues in lamin LIII is also of interest in another context. Studies with K-ras have shown that a polybasic stretch of six amino acid residues, although with a different order of lysine and arginine residues, functions as a secondary signal to target

Fig. 6. Phylogenetic relationship of vertebrate lamins. The rod domains of vertebrate lamins were aligned and submitted to phylogenetic analysis using the UPGMA (unweighted pair group with arithmetic mean method) method with the PAUP program version 40b2a (Swofford, 1998). Sequences are from man (Hs B1, P20700; Hs $\mathrm{B} 2$, the complete amino acid sequence of human lamin B2 was assembled from the partial cDNA sequence M94362 and the EST clone BE295831; Hs A, P02545), from chicken (Gg B1, P14731; Gg B2, P14732; Gg A, P13648), from Xenopus (X1 B1, P09010; X1 B2, P21910; Xl A, P11048; Xl LIII, P10999), from zebrafish (Dr B1, AJ250201; Dr B2, AJ005936; Dr A, this study AF397016; Dr LIII, this study AF397015), and from Caenorhabditis (Ce B, S42257) 
K-ras to the plasma membrane (Hancock et al., 1990; Roy et al., 2000).

The giant nuclei of amphibian and fish oocytes are characterized by several peculiarities. Their chromatin is not closely associated with the nuclear lamina as it is in somatic cells but is organized in lampbrush chromosomes located in the center of the nucleoplasm. These oocytes also stockpile large amounts of maternal RNA and protein. Their chromosomes are in a postreplication state and are highly engaged in transcription. Early cleavage nuclei, in contrast, are rapidly replicating but are transcriptionally silent (Newport and Kirschner, 1982b). With the exception of the newly replicated DNA these nuclei are assembled entirely of maternal components. It is thus suggestive to speculate that this dramatic change of nuclear function needs nuclear components with special functional adaptations. The specific synthesis of two variants of a particular lamin subtype that differ in their association with nuclear membranes might be one of these adaptations. Early development of mammals and birds proceeds with normal cell cycles. This might explain why lamin LIII is restricted to fish and amphibians. Future studies will focus on the functional analysis of the lamin LIII variants in oocyte and early cleavage nuclei.

Acknowledgements. We thank Holger Knaut, Max Planck Institute for Developmental Biology, Tübingen, Germany, for the zebrafish cDNA, Silke Prätzel and Lutz Langbein, German Cancer Research Center, Heidelberg, Germany, for help with the generation of antibodies, Andreas Hunziker, German Cancer Research Center, Heidelberg, Germany, for DNA sequencing, and Anton Karabinos, Max Planck Institute for Biophysical Chemistry, Göttingen, Germany, for help with the phylogenetic tree calculations. This work was supported by the Deutsche Forschungsgemeinschaft priority program "Functional architecture of the cell nucleus" (Sti 98/6-2).

\section{References}

Aebi, U., Cohn, J., Buhle, L., Gerace, L. (1986): The nuclear lamina is a meshwork of intermediate-type filaments. Nature 323, 560-564.

Altschul, S. F., Madden, T. L., Schaffer, A. A., Zhang, J., Zhang, Z., Miller, W., Lipman, D.J. (1997): Gapped BLAST and PSI-BLAST: a new generation of protein database search programs. Nucleic Acids Res. 25, 3389-3402.

Beck, L. A., Hosick, T. J., Sinensky, M. (1990): Isoprenylation is required for the processing of the lamin A precursor. J. Cell Biol. 110, $1489-1499$.

Benavente, R., Krohne, G., Franke, W. W. (1985): Cell type-specific expression of nuclear lamina proteins during development of Xenopus laevis. Cell 41, 177-190.

Döring, V., Stick, R. (1990): Gene structure of nuclear lamin LIII of Xenopus laevis; a model for the evolution of IF proteins from a laminlike ancestor. EMBO J. 9, 4073-4081.

Firmbach-Kraft, I., Stick, R. (1993): The role of CaaX-dependent modifications in membrane association of Xenopus nuclear lamin B3 during meiosis and the fate of B3 in transfected mitotic cells. J. Cell Biol. 123, $1661-1670$.

Fisher, D. Z., Chaudhary, N., Blobel, G. (1986): cDNA sequencing of nuclear lamins $\mathrm{A}$ and $\mathrm{C}$ reveals primary and secondary structural homology to intermediate filament proteins. Proc. Natl. Acad. Sci. USA 83, 6450-6454.

Fuchs, E., Weber, K. (1994): Intermediate filaments: structure, dynamics, function, and disease. Annu. Rev. Biochem. 63, 345-382.

Furukawa, K., Hotta, Y. (1993): cDNA cloning of a germ cell specific lamin B3 from mouse spermatocytes and analysis of its function by ectopic expression in somatic cells. EMBO J. 12, 97-106.
Furukawa, K., Inagaki, H., Hotta, Y. (1994): Identification and cloning of an mRNA coding for a germ cell-specific A-type lamin in mice. Exp. Cell Res. 212, 426-430.

Gerace, L., Blobel, G. (1980): The nuclear envelope lamina is reversibly depolymerized during mitosis. Cell 19, $277-287$.

Hancock, J. F., Paterson, H., Marshall, C. J. (1990): A polybasic domain or palmitoylation is required in addition to the CAAX motif to localize p21ras to the plasma membrane. Cell 63, 133-139.

Hausen, P., Riebesell, M. (1991): The early development of Xenopus laevis: an atlas of the histology. Springer, Berlin, New York.

Hausen, P., Wang, Y. H., Dreyer, C., Stick, R. (1985): Distribution of nuclear proteins during maturation of the Xenopus oocyte. J. Embryol. Exp. Morphol. 89, Suppl., 17-34.

Heald, R., McKeon, F. (1990): Mutations of phosphorylation sites in lamin A that prevent nuclear lamina disassembly in mitosis. Cell 61, $579-589$.

Hegele, R. A. (2000): The envelope, please: nuclear lamins and disease. Nature Med. 6, 136-137.

Hennekes, H., Nigg, E. A. (1994): The role of isoprenylation in membrane attachment of nuclear lamins. A single point mutation prevents proteolytic cleavage of the lamin A precursor and confers membrane binding properties. J. Cell Sci. 107, 1019-1029.

Hesse, M., Magin, T. M., Weber, K. (2001): Genes for intermediate filament proteins and the draft sequence of the human genome: novel keratin genes and a surprisingly high number of pseudogenes related to keratin genes 8 and 18. J. Cell Sci. 114, 2569-2575.

Hofemeister, H., Weber, K., Stick, R. (2000): Association of prenylated proteins with the plasma membrane and the inner nuclear membrane is mediated by the same membrane-targeting motifs. Mol. Biol. Cell 11, 3233-3246.

Holtz, D., Tanaka, R. A., Hartwig, J., McKeon, F. (1989): The CaaX motif of lamin A functions in conjunction with the nuclear localization signal to target assembly to the nuclear envelope. Cell 59, 969977.

Hutchison, C. J., Alvarez-Reyes, M., Vaughan, O. A. (2001): Lamins in disease: why do ubiquitously expressed nuclear envelope proteins give rise to tissue-specific disease phenotypes? J. Cell Sci. 114, 9-19.

Huttenlauch, I., Peck, R. K., Stick, R. (1998): Articulins and epiplasmins: two distinct classes of cytoskeletal proteins of the membrane skeleton in protists. J. Cell Sci. 111, 3367-3378.

Kane, D. A. (1999): Cell cycles and development in the embryonic zebrafish. Methods Cell Biol. 59, 11-26.

Kilic, F., Johnson, D. A., Sinensky, M. (1999): Subcellular localization and partial purification of prelamin A endoprotease: an enzyme which catalyzes the conversion of farnesylated prelamin A to mature lamin A. FEBS Lett. 450, 61 -65.

Kitten, G. T., Nigg, E. A. (1991): The CaaX motif is required for isoprenylation, carboxyl methylation, and nuclear membrane association of lamin B2. J. Cell Biol. 113, 13-23.

Krohne, G., Waizenegger, I., Höger, T. H. (1989): The conserved carboxy-terminal cysteine of nuclear lamins is essential for lamin association with the nuclear envelope. J. Cell Biol. 109, 2003-2011.

Lehner, C. F., Stick, R., Eppenberger, H. M., Nigg, E.A. (1987): Differential expression of nuclear lamin proteins during chicken development. J. Cell Biol. 105, 577-587.

Lenz-Böhme, B., Wismar, J., Fuchs, S., Reifegerste, R., Buchner, E., Betz, H., Schmitt, B. (1997): Insertional mutation of the Drosophila nuclear lamin Dm0 gene results in defective nuclear envelopes, clustering of nuclear pore complexes, and accumulation of annulate lamellae. J. Cell Biol. 137, 1001-1016.

Lin, F., Worman, H. J. (1993): Structural organization of the human gene encoding nuclear lamin A and nuclear lamin C. J. Biol. Chem. 268, $16321-16326$.

Lin, F., Worman, H. J. (1995): Structural organization of the human gene (LMNB1) encoding nuclear lamin B1. Genomics 27, 230-236.

Liu, J., Ben-Shahar, T. R., Riemer, D., Treinin, M., Spann, P., Weber, K., Fire, A., Gruenbaum, Y. (2000): Essential roles for Caenorhabditis elegans lamin gene in nuclear organization, cell cycle progression, and spatial organization of nuclear pore complexes. Mol. Biol. Cell 11, 3937-3947. 
Loewinger, L., McKeon, F. (1988): Mutations in the nuclear lamin proteins resulting in their aberrant assembly in the cytoplasm. EMBO J. 7, 2301-2309.

Lourim, D., Kempf, A., Krohne, G. (1996): Characterization and quantitation of three B-type lamins in Xenopus oocytes and eggs: increase of lamin LI protein synthesis during meiotic maturation. J. Cell Sci. 109, $1775-1785$.

Lourim, D., Krohne, G. (1993): Membrane-associated lamins in Xenopus egg extracts: identification of two vesicle populations. J. Cell Biol. 123, $501-512$.

Machiels, B. M., Zorenc, A. H., Endert, J. M., Kuijpers, H. J., van Eys, G. J., Ramaekers, F. C., Broers, J. L. (1996): An alternative splicing product of the lamin A/C gene lacks exon 10. J. Biol. Chem. 271, 9249-9253.

Newport, J., Kirschner, M. (1982a): A major developmental transition in early Xenopus embryos: I. characterization and timing of cellular changes at the midblastula stage. Cell 30, 675-686.

Newport, J., Kirschner, M. (1982b): A major developmental transition in early Xenopus embryos: II. Control of the onset of transcription. Cell 30, $687-696$.

Nigg, E. A., Kitten, G. T., Vorburger, K. (1992): Targeting lamin proteins to the nuclear envelope: the role of CaaX box modifications. Biochem. Soc. Trans. 20, 500-504.

Peter, M., Nakagawa, J., Dorée, M., Labbe, J. C., Nigg, E. A. (1990): In vitro disassembly of the nuclear lamina and $M$ phase-specific phosphorylation of lamins by cdc2 kinase. Cell 61, 591-602.

Riemer, D., Wang, J., Zimek, A., Swalla, B. J., Weber, K. (2000): Tunicates have unusual nuclear lamins with a large deletion in the carboxyterminal tail domain. Gene 255, 317-325.

Röber, R. A., Weber, K., Osborn, M. (1989): Differential timing of nuclear lamin $\mathrm{A} / \mathrm{C}$ expression in the various organs of the mouse embryo and the young animal: a developmental study. Development 105, 365-378.

Roy, M. O., Leventis, R., Silvius, J. R. (2000): Mutational and biochemical analysis of plasma membrane targeting mediated by the farnesylated, polybasic carboxy terminus of K-ras4B. Biochemistry 39, $8298-8307$.

Schnölzer, M., Alewood, P., Jones, A., Alewood, D., Kent, S. B. (1992): In situ neutralization in Boc-chemistry solid phase peptide synthesis. Rapid, high yield assembly of difficult sequences. Int. J. Pept. Protein Res. 40, 180-193.
Selman, K., Wallace, R. A., Sarka, A., Qi, X. (1993): Stages of oocyte development in the zebrafish, Brachydanio rerio. J. Morphol. 218, $203-224$.

Stewart, C., Burke, B. (1987): Teratocarcinoma stem cells and early mouse embryos contain only a single major lamin polypeptide closely resembling lamin B. Cell 51, 383-392.

Stick, R. (1992): The gene structure of Xenopus nuclear lamin A: a model for the evolution of A-type from B-type lamins by exon shuffling. Chromosoma 101, 566-574.

Stick, R., Angres, B., Lehner, C. F., Nigg, E. A. (1988): The fates of chicken nuclear lamin proteins during mitosis: evidence for a reversible redistribution of lamin $\mathrm{B} 2$ between inner nuclear membrane and elements of the endoplasmic reticulum. J. Cell Biol. 107, $397-406$.

Stick, R., Hausen, P. (1985): Changes in the nuclear lamina composition during early development of Xenopus laevis. Cell 41, 191-200.

Stick, R., Krohne, G. (1982): Immunological localization of the major architectural protein associated with the nuclear envelope of the Xenopus laevis oocyte. Exp. Cell Res. 138, 319-313.

Sullivan, T., Escalante-Alcalde, D., Bhatt, H., Anver, M., Bhat, N., Nagashima, K., Stewart, C. L., Burke, B. (1999): Loss of A-type lamin expression compromises nuclear envelope integrity leading to muscular dystrophy. J. Cell Biol. 147, 913-920.

Swofford, D. L. (1998): PAUP. Phylogenetic analysis using parsimony (and other methods), version 4. Sinauer, Sunderland, MA.

Weber, K., Plessmann, U., Traub, P. (1989): Maturation of nuclear lamin A involves a specific carboxy-terminal trimming, which removes the polyisoprenylation site from the precursor; implications for the structure of the nuclear lamina. FEBS Lett. 257, 411-414.

Westerfield, M. (1995): The zebrafish book. University of Oregon Press, Eugene, Oregon.

Wilson, K. L., Zastrow, M. S., Lee, K. K. (2001): Lamins and disease: insights into nuclear infrastructure. Cell 104, 647-650.

Wolin, S. L., Krohne, G., Kirschner, M. W. (1987): A new lamin in Xenopus somatic tissues displays strong homology to human lamin A. EMBO J. 6, 3809-3818.

Yamaguchi, A., Yamashita, M., Yoshikuni, M., Nagahama, Y. (2001): Identification and molecular cloning of germinal vesicle lamin B3 in goldfish (Carassius auratus) oocytes. Eur. J. Biochem. 268, 932-939.

Zewe, M., Höger, T. H., Fink, T., Lichter, P., Krohne, G., Franke, W. W. (1991): Gene structure and chromosomal localization of the murine lamin B2 gene. Eur. J. Cell Biol. 56, 342 - 350. 